\title{
Designing for Balance: Out There and In Here
}

\author{
Tim Coughlan \\ tim.coughlan@open.ac.uk \\ Anne Adams \\ Institute of Educational Technology \\ The Open University, Milton Keynes, MK7 6AA, UK \\ a.adams@open.ac.uk
}

Yvonne Rogers

y.rogers@open.ac.uk

\begin{abstract}
This paper describes the 'Out There and In Here' project, in which we explore the combined use of mobile technologies and staticindoor technologies to support novel forms of collaborative field trip learning. We are currently developing a system to support balanced collaboration between geology students 'Out There' in the field, and their peers located in a specially designed 'In Here'laboratory. Here we explain the background to the project, and describe data collected on perceptions of field learning in geology that is directing design. In particular, we discuss bringing the 'Out There' experience 'In Here', whilst also enhancing the field experience. This requires the concurrent development of technologies and activities, and balancing the control required for effective learning with scope for user creativity.
\end{abstract}

Collaboration, Education, Touch technologies, Mobile Learning.

\section{INTRODUCTION}

Through the 'Out There and In Here' project we are exploring how new technologies can support synchronous collaborative learning experiences in field trips between students 'Out There', and others based indoors. Fieldwork in higher education geology courses is being used as our initial test domain, and it is accepted that these activities significantly enhance the learning of geological concepts [e.g., 4]. However the benefits of field learning are currently difficult or impossible to achieve for all: Mobilityimpaired students may be unable to participate, while for others, financial costs can be prohibitive. Organizations can also be unwilling to tackle the complexities of delivering these experiences. It has also been found that mobile technologies can play important roles and alter practices in field learning $[2,3,6,8]$. This project is therefore exploring the potential for novel learning experiences that connect field-based students with others who do not have direct physical access to the field location.

Geology field trips encompass a range of data collection and analysis activities, with the common aim of better understanding the geological history and current state of a given site. These activities are commonly performed in groups, and involve learning to use a range of specialised tools and methods to then gather evidence and produce broader conclusions.

In this project, our approach is to design novel hybrid learning experiences supported by a combination of new technologies. The underling idea is to link students in the field with others situated indoors, supporting a diverse set of learning activities.
Through collaboration between both parties, some of the benefits of field activities can be achieved by students who are not physically 'Out There', while those students can be supported by their 'In Here' collaborators. An inspirational analogy is the 'Treasure Hunt' TV game show, where studiobased participants guided a 'skyrunner' over a voice link, searching for clues to lead them to their next location. We propose that having students work together in this interactional way offers new opportunities to synchronously combine field data gathering with research, analysis and reflection. As well as involving students who are unable to go to the site, it can enable more of the inquiry process to happen there and then, rather than field-based students having to hold back on analysis until a later date, away from the actual location $[6,8]$. The system should provide both situations with experiences that would otherwise be missed, or were previously nonexistent - for example collaborating across large distances to explore a vastly different site to the student's location, or a location where safety issues would prevent student visits.

\section{RELATED WORK}

One of the earliest projects exploring how new technologies could enhance collaborative learning during field trips was Ambient Wood (Rogers et al, 2005). Novel technologies were developed to facilitate collaborative scientific inquiry by school children. These included light and moisture probes, a PDA to display readings and present images and location data, walkie-talkies for communication with facilitators, and an 'Ambient Horn' that produced 
sounds related to local ecological processes. The project found that the design of the devices led to specific roles, and that as the activity required the use of all the devices, each student was valuable to their team [8], highlighting the importance of tight integration between collaborative activity and technology design.

More recently, the Personal Inquiry project (PI) has explored how mobile technologies can be used to support evidence-based inquiry learning in schools. The project has iteratively developed and evaluated software for mobile and desktop use. Design issues include combined support for individual, group and class interactions, the use of menus to guide students' learning of inquiry processes, and functionality for data collection and analysis that balances structure with space for students to perform work themselves. The project has found evidence that by providing the freedom for personally-meaningful learning activities to be developed using the system, students are more motivated in their learning activities [2].

The LilyPad project explored collaborative inquiry support for university students in an ongoing environmental restorationproject, where students worked with complex scientific data sets, tools and visualisations. PDAs supported both the collection and analysis of data, and also communication with peers. The project found that the role of instructors was changed by the addition of the PDA as an information resource, often meaning that facilitation of learning became as important as instruction [8]. These projects have considered field and class learning as distinct in time as well as space, although they have often blurred the lines between the types of activities usually performed in these contexts. In contrast the Remote Accessible Field Trips project (RAFT) explores how real-time remote collaboration occurs between field and class. The development of a RAFT activity includes the design of the tasks to be performed and the distribution of these between the students. Data gathering equipment is given to field team members, along with video conferencing and text chat support. Classroom-based learners can ask questions, provide information and analyse data while influencing the field team members in real time. The findings stress the importance of assigning well-defined roles, in order that teachers can manage the activity effectively and that all students feel engaged. Roles developed for the RAFT project include 'Scout', 'Data Gatherer' and 'Task Manager' [6].

Hence, the extent to which mobile technologies can extend learning experiences when used in situ depends on the way different kinds of digital information are communicated and manipulated during ongoing physical activities. The goal of our research is to investigate further the extent to which distributed collaborative learning activities can be facilitated by being able to interact with various forms of information and data using a mobile device in the field and shared technologies in the lab. A particular focus is the nature of the communication and coordination that takes place between physical and digital activities.

\section{STAGE 1: INITIAL DESIGN PROCESS}

Fieldwork adds a practical, hands-on element to learning across many subjects. It provides direct experiences of the world and provokes essential processes of learning by doing. Field learning activities are often performed in groups, so also provide an important socio-cultural context for learning to take place. In this section we discuss our pilot studies and how they will now be built upon, and also an initial analysis of data collected from geologists, which is being used to direct our design process.

\subsection{Pilots: Enabling Remote Activity (ERA)}

The OTIH project builds upon the Enabling Remote Activity (ERA) project, which piloted technologies to allow higher education students with physical disabilities to participate in geological field trip activities (see Figure 1). Students situatednearby in vehicles or at a distance in a lab worked with their peers contexts, particularly because the 'static' students had much to offer the 'mobile' students in terms of access to information as part of analysis activities [3].

The ERA project found it possible to support activities that were perceived by students as much more engaging and useful when compared to students only engaged in virtual fieldtrips. Equitable collaborative learning was possible across the mobile and static. contexts, particularly because the 'static' students had much to offer the 'mobile' students in terms of access to information as part of analysis activities [3].
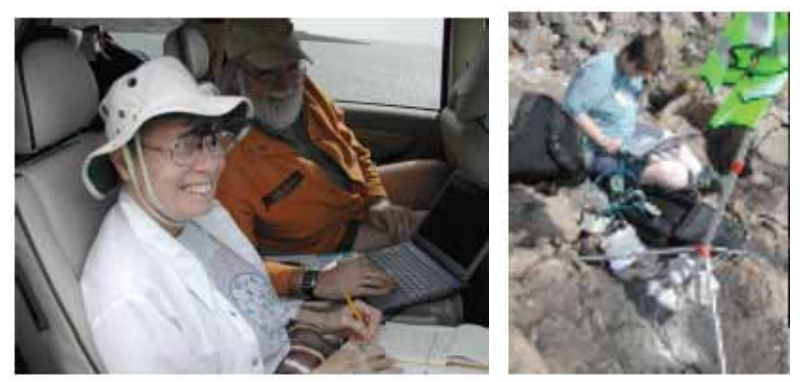

Figure 1: ERA Field Trials

\subsection{Moving Forward}

This project extends ERA and related work in several 
ways. Our plan is to enable multiple students 'In Here' to interact both with each other and with multiple students in the field through a combination of tabletop and mobile technologies. Through this we will explore how to support synchronous collaborative learning across diverse contexts, and with students communicating through diverse technologies. The aim is to improve the experiences of all students, but particularly to replicate as much as possible the learning benefits of the field experience for those students who are unable to be 'Out There'.

Whereas ERA used standard desktop and laptop PCs, the OTIH project is developing a 'Command and Control' style room to support the 'In Here' students in their work and to collaborate effectively with their field based peers, who will be equipped with smartphones amongst other technologies. Concurrently, we are working with educators to build an understanding of the activities that will be suited to this new learning context and the roles tutors might play in these activities. Both technologies and activities will be designed and evaluated iteratively through in-situ field trials.

\subsection{Capturing Geologists Perceptions}

The project aims to explore the possibilities for field trip learning that is 'replicated' for students who are not physically 'Out There'. This requires being sensitive to the experiences of both classes of students and their instructors. Building upon the practical knowhow developed through the ERA trials, we have elicited qualitative data from geologists on their perceptions of fieldwork. Firstly a set of posters were situated in a common room used by geology staff and postgraduates at the university. These asked for responses to the following questions:

1) "What is it about real fieldwork that makes it such a great learning experience?"

2) "What do students NEED to learn in the field?"

3) "What can students ONLY learn in the field?".

39 responses were thematically analysed into:

- Social and collaborative learning (e.g. "People, peers, discussion, combined learning journey")

- Immersion in the subject ("Concentrated. Progression of thought is focussed without distraction")
- Scale ("Context -from hand-lense scale to landscape scale")

- Senses ("All senses come into play (smell ( feel)")

- Seeing in reality ("It's different imagining rocks from books and pictures than seeing them in real life")

- Reality is complex, and not like the textbook ("That there's no such thing as a perfect example of a rock type").

- Learning the process from observation through analysis ("How to build up a 'story' from lots of observations").

- in the field through an ad-hoc network, supporting video links, voice over IP and file transfer directly from cameras and netbook computers at the site.

- 3-Dimensional Comprehension ("Relationships between hand sample, outcrop-scale and terrain scale").

- Essential practical skills like mapping, measurement and reporting ("How to locate features seen on a map in the real world and how to put observations onto a map").

\section{STAGE 2: DESIGN AND EVALUATION}

It is clear that delivering an effective experience requires interdependent consideration of technology and learning activities. In this section we describe the issues that are shaping the direction of the project and the activities that will take place.

\subsection{Technology Design}

The Out There and In Here system consists of two contexts and related technological support systems, linked over a network to provide scope for collaborative learning as follows:

\subsubsection{Out There}

The field-based students will be provided with equipment including smartphones, cameras and portable computers. Depending on the connectivity available at the site, ad-hoc wireless networks will be set up using 3G, broadband and / or satellite links to connect with the 'In Here' environment. Systems for video links, Voice over IP, and file transfer in 
field trip contexts were piloted in the ERA project. This project will now build upon these experiences to further develop interfaces for mobile collaboration using technologies such as GPS tracking.

\subsubsection{In Here}

In the 'In Here' laboratory, a multi-touch tabletop (Microsoft Surface [7]) will provide a central point for discussion amongst the In Here students and a window to enable collaboration with the field-based students. The system will make use of tangible object tagging to integrate relevant artefacts in to the interface. Table top screens are particularly suitable for interacting with spatial data [5] and in this case will provide a 'birds-eye' view of the site, as well as a place to review and discuss documents as a group. The Surface will support digital information sharing and referencing among the students, and will be complemented by additional computers and awareness displays to support research and to display data streams from the students 'Out There' and from static cameras on site. This will support an immersive shared experience, with tools tailored for expected roles.

\subsection{Activity Design}

As in RAFT [6], activities and roles will be developed through participatory processes. In particular, we must design to provoke and support the interactions and roles that are integral to field trip activities. To do this requires balancing scripted activities with room for improvisation led by students or instructors. It is clear from the research described previously that these activities should not simply replicate existing practice. Building upon our initial understanding of the field trip experience with iterative design and evaluation cycles, we need to identify the characteristics of activities that are suited to the Out There and In Here paradigm.

Designing these activities will require focusing on experiences where collaboration is necessary and the roles adopted are equitable and meaningful. It is expected that some roles will be assumed differently because of the diverse capabilities of students in each contexts, but that all students will be able to contribute data, and to suggest or comment on hypotheses about the history and nature of the site. The ERA project pilots identified that while those 'In Here' had better access to digital resources, bigger screens and faster computation for analysis, those 'Out There' had immersion in the actual environment, mobility and a sense of scale in their favour. This project seeks to bring these abilities and experiences closer together, rather than segregating students experiences to each side of the activity.

Previous research details how instructor roles can change when mobile technology is introduced [8]. Through workshops and interview data an understanding will be formed of how instructors currently perceive their role. The student roles used in the RAFT project were quite generic, while in this project roles will also reflect specialist geology field skills. The use of specialist tools (e.g. hand lenses, hammers and formal logs) will guide activities both 'In Here' and 'Out There' as objects that relate to particular tasks, reflective processes and expected outcomes.

In contrast to textbooks, fieldwork is a guided experience through the complex and dynamic nature of the world where students learn through engaging with contextual reality. The open-ended nature of inquiry in the real world should also lead to students' creation and ownership of aspects of the activities [2], which in turn supports individual and group identity development as geologists. Rather than being prescribed rigid activities, it is essential that instructors and students can create and direct their experiences whilst using the system. Beyond supporting interactions on the day, there should also be scope for reusing created content for reflection, reporting, and in subsequent field activities [6].

\subsection{Taking the 'Out There' to 'In Here'}

The themes in section 3 highlight challenges to providing a field-like experience to students 'In Here'. The development of a platform for social and collaborative interaction will utilise a range of communication channels, and consider how they support possibilities for dynamic social, vicarious and peer-assisted learning. Replicating field based immersion for 'In Here' students will require thinking about the experience holistically, and to designing activities and a context to take students away from their everyday lives, with significant dedicated time and space.

To stimulate a range of senses, and to project the reality of the field setting, we will make use of tangible objects in the 'In Here' system. For example, field tools used by geologists and rocksfrom the site could form part of the interface through object tagging and recognition. Effective use of field-based multimedia can bring the experience inside when, for example, variations in the sound a hammer makes on different surfaces provides important information to geologists. Scale is essential to geological understanding, with a focus on both minute details of grains in rocks and the whole landscape. 'In Here' support will be specifically designed to deliver scale information from 'Out There'. Pilot studies in the ERA project provided static camera viewpoints to complement detailed images received from mobile cameras. Map interfaces and Geographic Information Systems (GIS) can also be leveraged for this purpose. 


\subsection{Connections and Degradation}

One of the major issues with a complex networked system, particularly in mobile contexts, is the ability to deal with unpredictable losses of connection, degradation, and the differences in available bandwidth at different (commonly rural) sites. In response to this, we are seeking to understand how to develop graceful degradation whilst utilising the qualities of connections to provide the best collaborative learning experience. Questions include: How do high bandwidth channels such as video links provide a different or improved experience when compared to text chat and still images? If connections are degraded or lost completely, how can a system support the continuation of effective learning at both ends?

In designing the technology we will approach these issues by supporting a range of communications channels, and not requiring a consistent network link for the sub-systems at either end to function. Data will be synchronised when possible between local servers at each site, making it possible to access files produced earlier from the other site, even if the link is currently down or congested. In terms of the activity, it is important to provide a loose structure, including tasks that can be performed by both 'Out There' and 'In Here' sub-groups, and/or as a full combined group according to circumstances.

\subsection{Finding the Balance}

It is clearly necessary to design activities and technologies interdependently in order that we to deliver an effective user experience to each of the students involved. Whilst each system component is important, it is the balanced, overall experience of those using the system that is being designed and evaluated. For example it is important that we avoid producing drone-like mobile students, entirely directed by their static peers, or devices that detract from the outdoor experience.

One approach to achieving this is through utilising the conceptual framework of Trajectories developed by Benford et al [1]. This highlights the importance of hybrid structures, transitions, management and interleaving in developing canonical trajectories through an experience, and managing individual participants' variations from this. Examples from their research share many commonalities with our envisaged experiences, including collaborative interactions across varied roles, technologies and contexts. In this instance, pedagogical processes are central to developing individual, group and whole trajectories.

Orchestration processes are used to balance scripted control with scope for user improvisation. When designing a complex experience there can be a tendency towards restricting user control. This can be detrimental to the characteristics of good fieldwork where student led and tutor guided activities are neither idealised nor predictable. On the other hand, achieving learning outcomes is essential, and some predictability in trajectories through space (both learning and physical) and artefact usage can provide leverage for designers that benefit learners. The evaluations will require multiple data collection and analysis methods (observations, log \& video analysis and interviews) to uncover both individual and group activity trajectories. Foci for evaluation include the value of the system to provoke learning outcomes through collaborative inquiry, its ability to replicate aspects of field work for those students 'In Here' while providing a valuable experience for those 'Out There', and the suitability of the system from the point of view of instructors. All users should feel a sense of engagement, and of being of value to the group.

\section{CONCLUSIONS}

In this project it is essential to balance the concerns of those 'In Here' with those 'Out There' to provide an equitable learning experience. We also need to balance the utility of structure to assure certain expectations and outcomes, with space for student and instructor creativity. The project is expected to result in a range of valuable outcomes and understanding, which can be applied to wider educational, social and technological concerns.

\section{ACKNOWLEDGMENTS}

This research is funded by the EPSRC under grant $\mathrm{EP} / \mathrm{H} 022589 / 1$

\section{REFERENCES}

[1] Benford, S., Giannachi, G., Koleva, B. \& Rodden, T. 2009. From Interaction to Trajectories: Designing Coherent Journeys Through User Experiences, Proceedings of the ACM Conference on Human Factors in Computing Systems. ACM Press. 709718.

[2] Collins, T., Gaved, M., Mulholland, P., Kerawalla, C., Twiner, A., Scanlon, E., Jones, A., Littleton, K., Conole, G. \& Tosunoglu, C. 2008. Supporting location-based inquiry learning across school, field and home contexts, Proceedings of MLearn '08.

[3] Davies, S. \& Bartlett, J. 2007. Report for the Enabling Remote Activity project, JISC TechDis, available from http://www.techdis.ac.uk/index. php?p=2_1_7_25_13

[4] Elkins, J. T. \& Elkins, N. M. L. 2007. Teaching Geology in the Field: Significant Geoscience Concept Gains in Entirely Field-based Introductory Geology Courses, Journal of Geoscience Education, v. 55, n.2. NAGT. $126-132$. 
[5] Forlines, C. \& Shen, C. 2005. DTLens: Multi-user Tabletop Spatial Data Exploration, Proceedings of UIST '05. ACM Press. 119-122.

[6] Joel, S., Arnott, J.L., Hine, N., Ingvarsson, S., Rentoul, R. \& Schofield, S. 2004. A Framework for Analysing Interactivity in a Remote Access Field Exploration System. IEEE

International Conference on Systems, Man and Cybernetics. vol. 3. IEEE Press. 2669-2674.

[7] Microsoft Surface, http://www.microsoft.com/ surface/

[8] Rogers, Y. \& Price, S. 2008. The Role of Mobile Devices in Facilitating Collaborative Inquiry In Situ, Research and Practice in Technology Enhanced Learning. 3 (3), 209-229. 